\title{
Dynamical refinement: The way to improved fit, accurate structure models and absolute structure from 3D ED data. Lukas Palatinus ${ }^{1}$ \\ ${ }^{1}$ Institute of Physics of the Czech Academy of Sciences \\ palat@fzu.cz
}

\begin{abstract}
Three-dimensional electron diffraction (3D ED) is a method of collecting electron diffraction data from single micro- or nanocrystals without the need for special orientation of the crystals with respect to the primary beam. Since its first introduction by Kolb et al. in 2007 [1], it has been developed, improved and applied in many laboratories around the globe in several variations and under a number of names, including ADT, EDT, RED, MicroED, cRED etc. - see the review by Gemmi et al. [2] for an overview. One of the recurring problems in refining structure models against 3D ED data is the presence of dynamical diffraction effects. These effects are usually negligible in $\mathrm{x}$-ray or neutron diffraction, but they are strong and unavoidable in electron diffraction. In most applications, the dynamical effects are nevertheless ignored and so-called kinematical approximation is used. Although this approach is often good enough to provide the structure model, it results in significantly underfitted data, increased figures of merit (typical refinement R-values R1(obs) range between $15 \%$ and $25 \%$ instead of the $\sim 4 \%$ expected on the basis of experimental uncertainties of measured intensities) and loss of information about the fine details of the structure under investigation. It is possible to include the dynamical effects in the structure refinement from 3D ED data [3,4]. This procedure is called dynamical refinement. It has been shown to significantly improve the fit, typically to R1(obs) between $7 \%$ to $9 \%$, provide better sensitivity to fine structural details and weak scatterers including the hydrogen atoms [5] and allow the determination of absolute structure of non-centrosymmetric crystals $[4,6]$. No special data collection procedure is needed for the application of dynamical refinement, and it can be applied to all types of 3D ED data. However, special data processing is required. Such data processing is available in the software PETS2 [7]. The dynamical refinement method is available in the crystallographic computing system Jana2006 [8] and its successor Jana2020. As already mentioned, dynamical refinement provides a significantly better fit to the electron diffraction data. For example, the structure refinement of the natural zeolite natrolite resulted in $\mathrm{R} 1$ (obs) $=5.96 \%$, while the kinematical refinement yielded an (already very good) R1(obs)=11.9\%. The R1(obs) of the refinement of the organic molecule carbamazepine decreased from $16.4 \%$ for the kinematical refinement to $8.77 \%$ for the dynamical refinement. The decrease in the figures of merit is accompanied by the corresponding decrease of the noise in the difference potential maps, typically by a factor of 1.5 to 3 . This in turn allows seeing weaker signals, including, for example, partially occupied hydrogen atoms in the vicinity of heavier ions [5,9]. An important aspect is the ability of dynamical refinement to determine the absolute structure of non-centrosymmetric crystals and thus the absolute configuration of chiral molecules. The physical effect behind this ability is not the resonant scattering like for x-ray diffraction, but the multi-beam interference. Hence, the limitations of absolute structure determination inherent to x-ray diffraction do not apply to electron diffraction. The determination of absolute structure is very robust and can be based on a simple comparison of the figures of merit of the two inverted models. The difference in the R1 values is typically several percent. Unlike for x-rays, the absolute structure can be equally easily determined for heavy-atom structures and light-atom structures [4,6]. Although the dynamical refinement improves the fit significantly compared to the kinematical refinement, its current implementation still does not describe the electron diffraction data fully. To further improve the fit, it is necessary to properly account for further effects like crystal imperfections, effects of inelastic scattering and also the bonding effects in the electrostatic potential.
\end{abstract}

[1] Kolb U, Gorelik T, Kübel C, Otten MT, Hubert D. Ultramicroscopy 107, 507-13 (2007).

[2] Gemmi M, Mugnaioli E, Gorelik TE, Kolb U, Palatinus L, Boullay P, et al. ACS Cent. Sci. 5,1315-29 (2019).

[3] Palatinus L, Petřiček V, Corrêa CA. Acta Cryst. A. 71, 235-44 (2015).

[4] Palatinus L, Corrêa CA, Steciuk G, Jacob D, Roussel P, Boullay P, et al. Acta Cryst. B. 71, 740-51 (2015).

[5] Palatinus L, Brázda P, Boullay P, Perez O, Klementová M, Petit S, et al. Science 355, 166-9 (2017).

[6] Brázda P, Palatinus L, Babor M. Science 364, 667-9 (2017).

[7] Palatinus L, Brázda P, Jelinek M, Hrdá J, Steciuk G, Klementová M. Acta Cryst. B. 75, 512-22 (2019).

[8] Petřiček V, Dušek M, Palatinus L. Z. Krist. 229, 345-52 (2014).

[9] Steciuk G, Majzlan J, Plášil J. IUCrJ 8, 116-23 (2021). 